\title{
Could Grid Facilitate Demanding Watermarking Media Applications?
}

\author{
B. Vassiliadis ${ }^{1}$, V. Fotopoulos ${ }^{1}$, M. Xenos ${ }^{1,2}$, A. N. Skodras ${ }^{1,2}$ \\ ${ }^{1}$ Digital Systems and Media Computing Laboratory \\ School of Sciences and Technology, Hellenic Open University, \\ 13-15 Tsamadou st. GR- 26222, Patras, Greece \\ ${ }^{2}$ R.A. Computer Technology Institute, \\ 61 Riga Feraiou st., GR-26221, Patras, Greece \\ $\{b b, v f 1, x e n o s, s k o d r a s\} @ e a p . g r$
}

\begin{abstract}
Copyright protection is becoming an important issue for organisations that distribute digital content through e-learning applications. One of the most promising methods for copyright protection is digital watermarking, a highly demanding application in terms of CPU load. In this communication, some specific cases are presented where GRID computing could be used as an enabling/assisting technology for watermarking. Specific cases of heavy load mathematical transformations for large media objects are discussed. A proposal for incorporating the watermarking procedure in the Grid architecture is also briefly presented.
\end{abstract}

Keywords: E-learning, Grid, security, watermarking.

\section{INTRODUCTION}

E-learning is moving from the age of mass media to the age of knowledge media leading to the creation of new types of institutions and pedagogical models. Current e-learning systems include elements such as online communities and collaboration software delivering large amounts of on-line/off-line multimedia content. As content delivery services are gaining importance Intellectual Property Protection (IPR) is the new challenges for content management and distribution services.

The ease, with which perfect digital copies are produced, creates great concern to e-learning providers. Copyright violation represents a considerable source of financial loss. Achieving IPR is currently considered a strategic goal for many e-learning institutions. The two strategies proposed for achieving IPR for digital media include a priori protection (copy prevention) and posteriori protection (copy detection). Initially, IPR focused on security and encryption as a means of solving the issue of unauthorized copying. The shortcomings of CD/DVD copy prevention systems have shown the lack of effectiveness of the a priori protection [1]. A posteriori protection mechanisms, such as digital watermarking, are regarded as a valid solution for multimedia data in a networked environment [2].

A digital watermark is an invisible mark inserted in digital media such as digital images, video and audio so that it can be detected at a later stage as evidence of copyright or it can generally be used against any illegal attempt to either reproduce or manipulate the media. The delivery of large and/or digital objects through e-learning distribution channels and the ever increasing use of streaming media is making the need for efficient watermarking methods more demanding [3].

This paper proposes the use of Grid technology for watermarking large digital objects (video sequences, high resolution \& multi-spectral images e.t.c.) in large scale e-learning applications. This extremely demanding processing may become both feasible and operational within the Grid technology framework. Section 2 describes current trends and requirements for IPR in the e-learning domain and discusses the possibility of embedding a watermarking module in the fabric layer of the Grid. Section 3 presents an overview of media watermarking techniques and a series of watermarking cases in which the Grid could be used. Finally, section 5 presents future research directions and potentials.

\section{IPR IN LARGE SCALE E-LEARNING APPLICATIONS}

E-learning refers to all processes, activities and technologies that support learning through the use of information and communication technology. Large scale e-learning applications include the provision of large amount of elearning, multimedia material from organisations such as Open Universities or coalitions of educational organisations to thousands of students using on-line and off-line delivery. The use of wide distribution channels 
such as the Internet has forced e-learning organisations to set the protection of IPR as a strategic goal. In practice IPR most often refers to ownership rights of individuals, including copyrights, patents and trademarks. Attribution, that is the right to be recognized as an author or contributor, also falls in this category [12].

IPR for digital content, including e-learning multimedia, has received a lot of attention from the scientific communities in recent years. IPR standardization is now occurring in a number of organizations: the OpenEBook Forum [10] and the MPEG group are researching solutions in the e-book and multimedia sectors. The Internet Engineering Task Force (IETF) and the World Wide Web Consortium (W3C) have also investigated current trends in IPR and Digital Rights Management [11].

Future e-learning models will arise from the participation of Universities in highly dynamic partnerships established by the use of Grid networks. Maturing digital-rights-management, digital-signature, and watermarking technologies are linking with Grid and peer to peer (P2P) applications to enable legal and secure distributed networks and peer exchanges. For example large-scale availability of e-learning data over the net may also make necessary watermarking to keep track of source and owner. Future Grid-based media applications are viable alternatives and complements to existing systems for both creators/distributors and consumers.

Many research efforts in e-learning workflow modelling have recognized the view that such networked organizations will be integrated primarily by shared knowledge, not by tools or currently fragmented e-learning processes alone [13]. E-learning Grid portals, acting as service gateways, may integrate resources from multiple organizations, each with their own policies and mechanisms possibly multiple quality of service dimensions, including security, reliability, and performance. Security and privacy of sensitive digital data is an obvious requirement: authentication, data integrity, access control and data confidentiality are some of the most critical issues that must be dealt with in such infrastructures.

In the e-learning domain, IPR solutions need to be implemented in more comprehensive and sophisticated ways by incorporating [7]:

- $\quad$ support for the e-learning community not just as users but also as creators and owners of copyrightprotected content,

- IPR support throughout the life cycle of a digital resource,

- management of the interactive and dynamic nature of e-learning content.

In the near future there is the possibility of embedding watermarking modules in the fabric layer of the Grid architecture. Such a module would be based on the following two procedures:

- information watermarking,

- $\quad$ extraction or detection of the watermark.

Watermarks, combined with digital signature methods, may contain information about proprietary, copyright, the author, the user, the number of copies and other important information. Such a watermarking system should be robust, not ambiguous, invisible and difficult to remove. It would be useful to consider three different modes in such a procedure. In each mode, different digital objects may be processed: hypertext, image or audio/video objects. A control module identifies object type and the combination of methods to be used. It must be noted that at most 2-3 watermarks can be embedded in an image or video object, before the quality of reproduction is significantly altered. In order to maintain a high Quality of Service, a consensus must be found between multiple watermarking and its perceptibility in the digital object. Multiple watermarks can be inserted at the production level, for the identification of the distribution path and/or to identify the end-user path.

Watermarking can also be used for multimedia content identification. Digimarc's MediaBridge Reader uses the concept of "smart images" wherein the watermarked message includes pointers to some knowledge structure on a local database or on the Internet [9]. The watermark (message) detector extracts the message and hence extracts the additional information from the database. Such a method is also computationally expensive. Similar multimedia content identification modules could be embedded in the Collective layer of the Grid.

\section{MEDIA WATERMARKING TECHNIQUES}

Watermarking stands for the embedding of perceptually invisible information into digital data that identify the rightful creator/owner. Watermarks can be used to prevent illegal use, copy or manipulation of the digital content, as proof of ownership or tampering and others. The problem that these techniques have to encounter is the robustness of the watermark against common processing tasks. Any attempt to remove the ownership information from the original image is called an attack. Some common attacks for still images include filtering, JPEG compression, histogram modification, cropping, rotation and downscaling.

Image watermarking techniques can generally be divided in two main groups depending on the processing domain of host image that the watermark is embedded in. One is the spatial domain group of techniques, according to which the intensity values of a selected group of pixels are modified. The other is the frequency domain group, where a group of the transform coefficients of the image are altered. Up to date, frequency domain approaches have been proved more successful for image watermarking. The transforms usually employed are the discrete versions of the Fourier, Cosine and Wavelet transform (DCT, DFT and DWT) [4,5]. In these schemes, the image is being transformed via one of the aforementioned frequency transforms and watermarking is performed by altering 
the resulting transform coefficients of the image. Figure 1 presents the NxN DCT transformation and the corresponding algorithm in C.

The complexity of the Discrete Fourier and the Cosine transform is $\mathrm{O}(\mathrm{N} \log \mathrm{N})$ while for the Wavelet transform it is $\mathrm{O}(\mathrm{N})$. For large values of $\mathrm{N}$, these transformations are becoming extremely demanding in terms of $\mathrm{CPU}$ cycles; however respective algorithms are suitable for distributed processing, as demonstrated hereinafter.

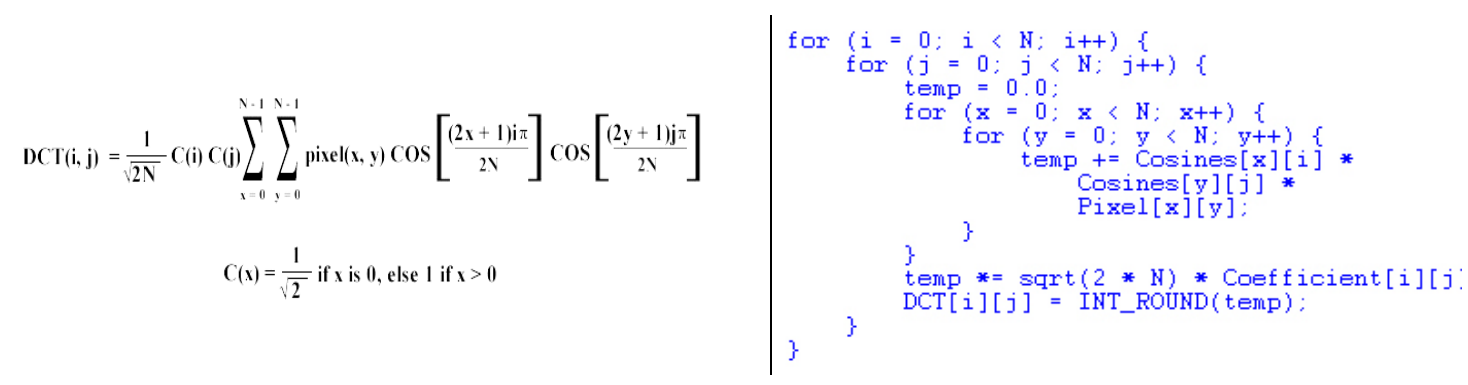

FIGURE 1: DCT transformation for a NxN pixels image and corresponding C code.

Another question that occurs in such approaches is the number and the position of the altered coefficients set in the frequency representation of the image. Many different ideas have been proposed, however methods that process the image as a whole are popular. In such cases the number of coefficients altered is in the order of a few thousands (e.g. 3-15 for the case of a $512 \times 512$ image).

Where Subband DCT has been used [6], a combination of both wavelets (Haar) and the Discrete Cosine Transform (DCT) has been employed. The original image is filtered and subsampled by means of a set of highpass and lowpass filters in order to produce a first level of decomposition. For each of the resulting four bands, DCT follows. After a zig-zag formulation to produce a one dimensional vector for each band, the standard multiplicative watermarking rule is applied to these coefficients:

$$
\mathrm{t}_{\mathrm{i}}^{\prime}=\mathrm{t}_{\mathrm{i}}+\mathrm{a} \mathrm{t}_{\mathrm{i}} \mathrm{x}_{\mathrm{i}} \text { with } i \in[\mathrm{L}, \mathrm{L}+\mathrm{M}-1]
$$

where $\boldsymbol{t}_{\boldsymbol{i}}$ are the transformed coefficients, $\boldsymbol{t}_{\boldsymbol{i}}$ ' are the watermarked coefficients and $\boldsymbol{x}_{\boldsymbol{i}}$ is a random sequence of Gaussian distribution, used as the watermark. The seed of the random sequence is the watermarking key of the system. The a-parameter has to do with the strength of the casting. The $i$ index ranges from $L$ to $L+M-1$. $L$ corresponds to the starting position in the coefficients set while $\boldsymbol{M}$ represents the number of those selected. For typical values of $L=5000$ and $M=20000$ (512x512 sized image), that means that a number of 80000 coefficients will be altered (20000 coefficients in each band). The whole scheme is graphically presented in Figure 2.

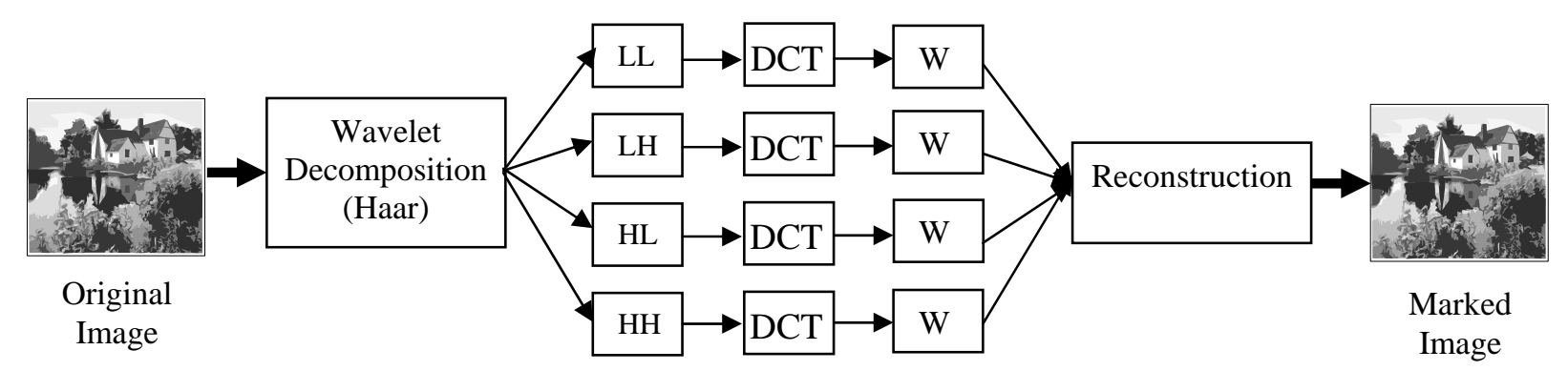

FIGURE 2: Subband DCT watermarking process

The question that almost naturally arises is how a Grid could assist this process. It is apparent from the above figure that four processes can be running at the same time. So, right after the wavelet decomposition, each of the four bands can be fed by the initial node (the one that did the decomposition) to one of four other nodes for individual processing (DCT and marking). One may also notice that four bands correspond to each colour channel. In RGB colour images, 12 nodes can be fed simultaneously, while for multispectral images, 4xN nodes can be used (let $\mathrm{N}$ be the number of bands). Additional decomposition levels can also be used allowing for more subbands per colour band and additional nodes. Even the decomposition/reconstruction stages may be parallelized since these stages, just like the transforms, can be performed with a row-column strategy. Thus, by loading the Grid nodes with different rows and columns, further parallelization is achievable.

As already mentioned, processing large digital objects such as large images or video is also CPU demanding. In order to decrease the huge memory requirements and to take advantage of the different nodes available in the Grid, a load distribution strategy can be used. This technique is based on dividing a digital object into smaller entities and processing in parallel on different Grid nodes. In the video case, instead of image tiles, individual frames are distributed to the watermarking nodes. The distribution process itself depends on the nature of the digital object. For example, in the case of still images, each image component is divided into several tiles, while in 
the case of video the process is frame wise, meaning that each node can process a bunch of frames. This video object division also depends on the format used i.e. different frames must be used for AVI and MPEG4 video processing. This allows different setups of Grid nodes, depending on the type of the medium being processed.

By generalizing, we will call this distribution strategy, 'tiling' since breaking a video object into frames is similar to dividing the image in smaller tiles. Tiling allows breaking up a single media processing request into multiple requests. This feature has the dual benefit of allowing data to be manipulated more efficiently between input/output operations and in memory and permitting the processing to be distributed to all available nodes. The immediate results are reduced memory overhead and processing time, and maximized application throughput. In short, this approach combines three major advantages: the parallelization of the I/O operation, the parallel processing of tiles and the distribution of computations on different processors. Eventually if the tile size is fixed, memory requirements may stay constant.

Image tiling has already been proposed for supporting image processing applications running on a single parallel machine [3]. For example parallel Processing Watermarking Embedding Schemes have demonstrated to be efficient from a computational and memory usage point of view for very large images. These schemes consist in dividing the image into tiles and watermarking each independently. Nevertheless, widely used single parallel machines usually have a small number of processors and are thus not as efficient in supporting processing of collections of large images or even video. However, research in parallel image processing has stressed the need for using Grid technology especially in demanding, dynamic environments.

\section{CONCLUSIONS}

New e-learning models based on shared knowledge may rely on the Grid infrastructure in order to deliver timely and on demand services. The extensive use of digital media in such applications increases security requirements. Watermarking, a computationally demanding method, is a promising solution for securing digital media in networked environments. In this work, the potential of using the Grid for enabling watermarking in e-learning environments has been exhibited. As watermarking technology is becoming mature, its combination with signature methods could be used for enhancing security and content identification and Grid could highly facilitate such media applications.

Future work in this field includes the identification of the different requirements for production and contribution, the requirement for registration authorities and the effective combination of watermarking and signature methods for effective content copyrighting. New media standards such as JPEG2000 and MPEG7 also offer much new potential. In this context, watermarking may prove to be a critical tool for broadcasters and providers of digital content. Besides e-learning, digital heritage [8] and digital television are just some of the applications that could make use of this technology in order to enhance security.

\section{REFERENCES}

[1] Fernandez, M. and Soriano, M. (2003), Intellectual Property Protection of e-Learning Contents. International Conference on Network Universities and e-Learning.

[2] Voyatzis, G. and Pitas, I. (2000), Image Watermarking for Copyright Protection and Authentication. In Bovik, A. (ed), Handbook of Image \& Video Processing, Academic Press.

[3] Debes, E. Dardier, G., Ebrahimi, T. and Herrigel, A. (2001) Watermarking Scheme for Large Images Using Parallel Processing. Proceedings of the SPIE and IS\&T Conference on Security and Watermarking of Multimedia Contents III.

[4] Cox, J. K., Thompson, F. and Shamoon, T. (1997) Secure Spread Spectrum Watermarking for Multimedia, IEEE Trans. On Image Processing, 6 (12).

[5] Fotopoulos, V. and Skodras, A.N. (2003) Digital Image Watermarking: An Overview. EURASIP Newsletter, 14 (4).

[6] Fotopoulos, V. and Skodras, A.N. (2000) A Subband DCT Approach to Image Watermarking. X European Signal Processing Conference.

[7] Xenos, M. and Skodras, A.N. (2003) Evolving from a Traditional Distance Learning Model to e-Learning. 2nd Workshop LeGE-WG: Towards a European Learning Grid Infrastructure - formulating the requirements of a European GRID for eLearning.

[8] Cheveau, L. (2002) Choosing a Watermarking System for Digital Television - The Technology and the Compromises, European Broadcasting Union, Switzerland IBC.

[9] Alattar, M. (2000) Bridging Printed Media and the Internet via Digimarc's Watermarking Technology. Multimedia and Security Workshop, ACM MM.

[10] Iannella, R. (2001) Digital Rights Management (DRM) Architectures. D-Lib Magazine, June 2001, 7(6), ISSN $1082-9873$.

[11] W3C (2001), Workshop on Digital Rights Management for the Web. http://www.w3.org/2000/12/drm-ws/

[12] AIMIA TR (2002) A Guide to Digital Rights Management. Australian Interactive Multimedia Industry Association.

[13] Hoppe, G. and Breitner, MH. (2003) Business Models for E-Learning. IWI Discussion Paper Series No2, http://econpapers.hhs.se/paper/handpaper/dp-287.htm. 